\title{
Oxidative stress causes Alu RNA accumulation via PIWIL4 sequestration into stress granules
}

\author{
Yeo Eun Hwang, Yu Mi Baek, Ahruem Baek \& Dong-Eun Kim ${ }^{*}$ \\ Department of Bioscience and Biotechnology, Konkuk University, Seoul 05029, Korea
}

\begin{abstract}
The Alu element, the most abundant transposable element, is transcribed to Alu RNA. We hypothesized that the PIWI protein regulates the expression of $A / u$ RNA in retinal pigment epithelial (RPE) cells, where accumulated Alu RNA leads to macular degeneration. Alu transcription was induced in RPE cells treated with $\mathrm{H}_{2} \mathrm{O}_{2}$. At an early stage of oxidative stress, PIWIL4 was translocated into the nucleus; however, subsequently it was sequestered into cytoplasmic stress granules, resulting in the accumulation of Alu RNA. An elevated amount of $A$ lu RNA was positively correlated with the disruption of the epithelial features of RPE via induction of mesenchymal transition. Therefore, we suggest that oxidative stress causes Alu RNA accumulation via PIWIL4 sequestration into the cytoplasmic stress granules. [BMB Reports 2019; 52(3): 196-201]
\end{abstract}

\section{INTRODUCTION}

Transposable elements (TEs) account for $\sim 45 \%$ of the human genome, which is a remarkable contribution compared to protein-coding genes that comprise only $1.5 \%$ of the human genome (1). Among the transposable elements, most of the mobile elements are retrotransposons that utilize the reverse transcription process. Retrotransposons duplicate themselves by the "copy and paste" process, in which retrotransposons are transcribed into RNA intermediates, reverse-transcribed to DNAs, and then inserted back into a new genomic position (2). Alu elements are one of the most abundant retrotransposable mobile elements that are present at more than one million copies in the human genome, which comprises $10 \%$ of the genome (1).

Alu elements have been suggested to play a role as a "necessary junk" factor that leads to evolutionary diversity of

*Corresponding author. Tel: +82-2-2049-6062; Fax: +82-2-34366062; E-mail: kimde@konkuk.ac.kr

https://doi.org/10.5483/BMBRep.2019.52.3.146

Received 4 July 2018, Revised 27 July 2018, Accepted 30 July 2018

Keywords: Alu RNA, Oxidative stress, Piwi-like protein 4 (PIWIL4), Stress granule the human genome $(3,4)$. However, abnormal regulation of their retrotransposon activities often leads to many diseases (5). The Human Genetic Mutation Database illustrated that Alu elements are associated with approximately $0.1 \%$ of human genetic diseases (5), including neurofibromatosis (6), leukemia (7), Alzheimer's disease (8), and breast cancer (9). These diseases are caused by inappropriate insertions of $A l u$ elements in the coding regions of the respective causative genes. Besides this, excessive amounts of transcription products, such as Alu RNAs, have been reported to elicit toxicity in cells (10).

Alu RNA transcribed from the Alu element by RNA polymerase III is a $\sim 300$ nucleotide long non-coding RNA containing both single- and double-stranded regions. Despite the huge amounts of Alu elements, they are usually transcribed into Alu RNAs at very low levels $\left(10^{2}-10^{3}\right.$ molecules/cell) due to their weak promoter (11). However, Alu RNA expression is elevated under various stress conditions, such as heat shock, protein synthesis inhibition, and viral infection (10, 11). Recent studies have suggested the accumulation of Alu RNA as a pathological causative factor of age-related macular degeneration (AMD), which is one of the major eye diseases that results in blindness. Deficiencies in the RNA processing enzyme DICER1 has been suggested to cause Alu RNA accumulation in the retina, which then triggers NLRP3 inflammasome activation, resulting in the degeneration of retinal cells $(12,13)$. In addition to AMD, excessive amounts of Alu RNA have been also reported in colorectal cancer cells, in which tumor progression have been correlated with Alu RNA amounts. Alu RNA has been observed to sequester microRNA (i.e. miR-566), which led to epithelial-to-mesenchymal transition (EMT) in the cancer cells (14). Although augmented Alu RNA amounts have been suggested due to DICER1 deficiency in an AMD model mouse, the relationship between DICER1 expression and Alu RNA transcript level was not correlated in tissues from patients with colorectal cancer (14). Thus, it is necessary to further investigate whether Alu RNA accumulation is caused by a lack of RNA processing by DICER1 and/or elevated transcription of Alu elements, which causes the same increase of endogenous Alu transcripts.

P-element induced wimpy testis in Drosophila (PIWI), a member of the Argonaute protein family, is an RNA-based genetic immune system that represses the invasion of retro-

ISSN: 1976-670X (electronic edition)

Copyright (c) 2019 by the The Korean Society for Biochemistry and Molecular Biology

(c) This is an open-access article distributed under the terms of the Creative Commons Attribution Non-Commercial License (http://creativecommons.org/licenses/by-nc/4.0) which permits unrestricted non-commercial use, distribution, and reproduction in any medium, provided the original work is properly cited. 
transposons, including Alu elements (15). PIWI specifically binds to PIWl-interacting RNAs (piRNAs), which are different from miRNAs and siRNAs because piRNAs (26-31 nts in length) are not processed by DICER1. When PIWI/piRNA complexes are formed, they translocate into the nucleus and epigenetically diminish retrotransposon expression via heterochromatin formation to maintain genomic stability (16). Although PIWI proteins mainly play a role in protecting the genome in germ cells, other functions of PIWI have also been identified in somatic cells. Various types of tumors, including breast, cervical, and colorectal cancer showed overexpression of piwi genes (17). Increased amounts of PIWIL4, a PIWI-like protein in humans, inhibited the p14ARF/p53 apoptosis pathway, which led to the proliferation of cervical cancer cells (18). Moreover, mRNA and protein expression levels of PIWIL1 was highly upregulated in hepatocellular carcinoma patients, while depletion of PIWIL1 in hepatocellular carcinoma cells reduced the cellular proliferation rate (19). In addition to their expression in cancer cells, PIWI-like proteins were also detected in human ocular tissues. It has been observed that knockdown of PIWI-like protein 4 (HIWI2) disrupted the expression and localization of tight junctions in retinal epithelial cells (20). Loss of tight junctions is pathologic to retinal epithelial tissues since it causes a collapse of the barrier against new blood vessels (21). However, the relationship between PIWI proteins and the levels of Alu RNAs in oxidative stress-inflicted retinal epithelial cells and its subsequent pathology have not been fully elucidated.

Herein, we report that sequestration of PIWI proteins is a possible cause for the abundance of $A / u$ RNA in retinal pigment epithelial (RPE) cells under oxidative stress. We observed that PIWIL4 was co-localized with stress granules in $\mathrm{H}_{2} \mathrm{O}_{2}$-treated RPE cells with upregulated expression of $\mathrm{Alu}$ RNAs. When PIWIL4 was translocated into the nucleus through the inhibition of stress granule formation, the amounts of Alu transcripts were decreased. Accumulation of Alu RNAs was correlated with a disruption of tight junction in retinal epithelial cells under oxidative stress. Based on these results, we suggest that sequestration of PIWIL4 into the cytoplasmic stress granule impedes silencing of Alu RNA transcription, leading to an increase of Alu RNA in RPE cells under oxidative stress.

\section{RESULTS AND DISCUSSION}

\section{Oxidative stress induces $A / u$ transcription in retinal pigment epithelial cells}

It has been reported that various stress conditions, such as heat shock and viral infection, increased the levels of Alu RNAs in HeLa and K562 cells (10). We thus hypothesized that the amount of Alu RNA may increase in retinal epithelial cells under oxidative stress conditions. Retinal pigment epithelial cells (ARPE-19) were treated with various concentrations of $\mathrm{H}_{2} \mathrm{O}_{2}$ ranging from $19 \mu \mathrm{M}$ to $300 \mu \mathrm{M}$ for $24 \mathrm{~h}$. The amount of
Alu RNA was increased as the concentration of $\mathrm{H}_{2} \mathrm{O}_{2}$ increased (Fig. 1A). A $300 \mu \mathrm{M} \mathrm{H}_{2} \mathrm{O}_{2}$ treatment increased Alu RNA by 2 -fold in ARPE-19 cells, and this concentration was used for further experiments. Oxygen radicals were generated in cultured ARPE- 19 cells exposed to $\mathrm{H}_{2} \mathrm{O}_{2}$ for $24 \mathrm{~h}$, as seen by monitoring the increase in $2^{\prime}, 7^{\prime}$-dichlorofluorescin diacetate (DCFDA) fluorescence in cells (Fig. S1). We next investigated levels of Alu genomic DNA copies and transcripts in cultured ARPE-19 cells exposed to $\mathrm{H}_{2} \mathrm{O}_{2}$ at increasing durations up to $24 \mathrm{~h}$. Prolonged exposure to $\mathrm{H}_{2} \mathrm{O}_{2}$ for $24 \mathrm{~h}$ resulted in a 2-fold accumulation of Alu RNA compared to untreated cells, while a significant decrease of Alu RNA was observed with a short-term $\mathrm{H}_{2} \mathrm{O}_{2}$ treatment for $6 \mathrm{~h}$. Since Alu transcripts are known to induce the insertion of $A l u$ elements via retrotransposition, we quantified the amount of Alu genomic DNA copies. ARPE-19 cells exposed to $\mathrm{H}_{2} \mathrm{O}_{2}$ for $24 \mathrm{~h}$ did not show significant changes in the levels of Alu genomic DNA (Fig. 1B). These results indicate that RPE cells under oxidative stress undergo changes in Alu transcripts levels, with a decrease and increase in the early and late phases of ROS exposure, respectively, without altering Alu genomic DNA amounts.

Subsequently, we investigated the amounts of several factors
A

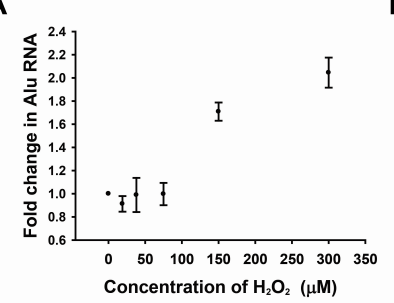

C

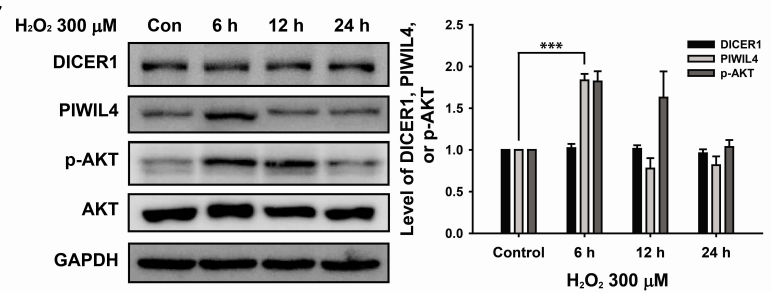

Fig. 1. Alu RNA expression was induced in retinal pigment epithelial (RPE) cells under oxidative stress. (A) Relative amount of Alu RNA was measured by real-time PCR in ARPE-19 cells treated with various concentrations of $\mathrm{H}_{2} \mathrm{O}_{2}(0,19,38,75,150$, and $300 \mu \mathrm{M}$ ) for $24 \mathrm{~h}$. (B) Total RNA and genomic DNA were obtained from ARPE-19 cells treated with $300 \mu \mathrm{M} \mathrm{H}_{2} \mathrm{O}_{2}$ for 6 , 12 , and $24 \mathrm{~h}$. Alu RNA and gDNA were quantified by real-time PCR, which was normalized to the amount of Alu RNA and gDNA in control cells. (C) Western blot analysis of DICER1, PIWIL4, AKT, and its phosphorylated form (p-AKT) in ARPE-19 cells treated with $\mathrm{H}_{2} \mathrm{O}_{2}(300 \mu \mathrm{M})$ for 6,12 , and $24 \mathrm{~h}$. Bar graph represents protein level normalized to GAPDH in the western blot images. Data are presented as the \pm S.E.M. $n=3$. $* P<$ $0.05, * * * \mathrm{P}<0.001$. 
such as DICER1, PIWIL4, AKT, and its phosphorylated form (p-AKT), which might affect Alu RNA accumulation under oxidative stress (Fig. 1C). Although a deficiency of DICER1 has been reported to be responsible for the accumulation of $\mathrm{Alu}$ RNA in the retinal epithelium $(12,13)$, we were not able to detect any significant changes in the level of DICER1 in cultured ARPE-19 cells exposed to $\mathrm{H}_{2} \mathrm{O}_{2}$ for 24 h. In contrast, the expression level of PIWIL4, which is likely involved in the suppression of retrotransposons, was transiently enhanced in the early phase of ROS exposure, which then diminished to control levels after $6 \mathrm{~h}$. Similar to the transient enhancement of PIWIL4 expression, AKT-phosphorylation was altered in a time-dependent manner, in which the phosphorylated form of AKT increased and decreased during the early (6 and $12 \mathrm{~h}$ ) and late $(24 \mathrm{~h})$ phases of ROS exposure, respectively. The PI3K/AKT pathway, which promotes cell survival and growth, has been known to be activated to cope with oxidative stress in cells $(22,23)$. Consistent with this result, we previously observed ROS-induced activation of the AKT/mTOR pathway in RPE cells under oxidative stress for 2-12 h (24). Based on these results, we suggest that RPE cells under oxidative stress display changes in Alu transcripts levels, which is concomitantly correlated with the expression level of PIWIL4 and AKT-phosphorylation in the early phase of ROS exposure. However, augmentation of Alu RNA expression in the late phase of ROS exposure was not related with the expression of Alu RNA generation nor processing factors such as PIWI-like protein and DICER1.

\section{PIWIL4 is translocated into the nucleus as an immediate response to the oxidative stress}

Since PIWIL4 has been known to silence the transcription of genetic elements in the nucleus, we investigated whether PIWIL4 proteins were responsible for the downregulation of Alu transcripts levels in the nucleus during the early phase of ROS exposure. The presence of PIWIL4 in the cytoplasm and nucleus was assessed by western blot analysis of two fractions containing cytosolic $\alpha \beta$-tubulin and nuclear p84 marker proteins (Fig. 2A). As compared with the amount of PIWIL4 without ROS treatment (control lanes), cytosolic PIWIL4 decreased and nuclear PIWIL4 increased at $6 \mathrm{~h}$ of $\mathrm{H}_{2} \mathrm{O}_{2}$ treatment. Furthermore, immunocytochemistry results showed that PIWIL4 was mainly located in the nucleus under the $\mathrm{H}_{2} \mathrm{O}_{2}$ treatment for $6 \mathrm{~h}$ (Fig. 2B), which was consistent with the western blot analysis of PIWIL4 enrichment in the nucleus. Based on these results, we inferred that a decrease of Alu transcript levels during the early phase of ROS exposure was ascribed to the downregulation of Alu transcription by the PIWIL4-enriched nucleus as an immediate response to the oxidative stress.

We also noticed that subcellular localization of fluorescently labeled PIWIL4 was juxtaposed onto the mitochondria in the cytoplasm prior to exposure to ROS (Fig. 2C). It has been previously demonstrated that piRNAs associated with PIWI proteins are processed by the endonuclease Zucchini (Zuc), which is present in the outer membrane of mitochondria (25). Thus, PIWIL4 proteins associated with piRNAs are mainly located near the mitochondria in the cytoplasm during quiescence without oxidative stress.

\section{PIWIL4 is sequestrated into stress granules under prolonged} oxidative stress conditions

When cells are exposed to stress, such as heat, oxidation, and viral infection, they form large cytoplasmic foci that are called as stress granules (SGs). By accumulating RNAs and RNA-binding proteins (RBPs) into granules, cells halt protein synthesis and protect important RNAs from extraneous stresses (26). Despite its cytoprotective role through ribostasis, prolonged formation of SGs may evoke cell death by hindering translation of proteins that are necessary for cell survival (27). A previous study reported that Ago2, an Argonaute family

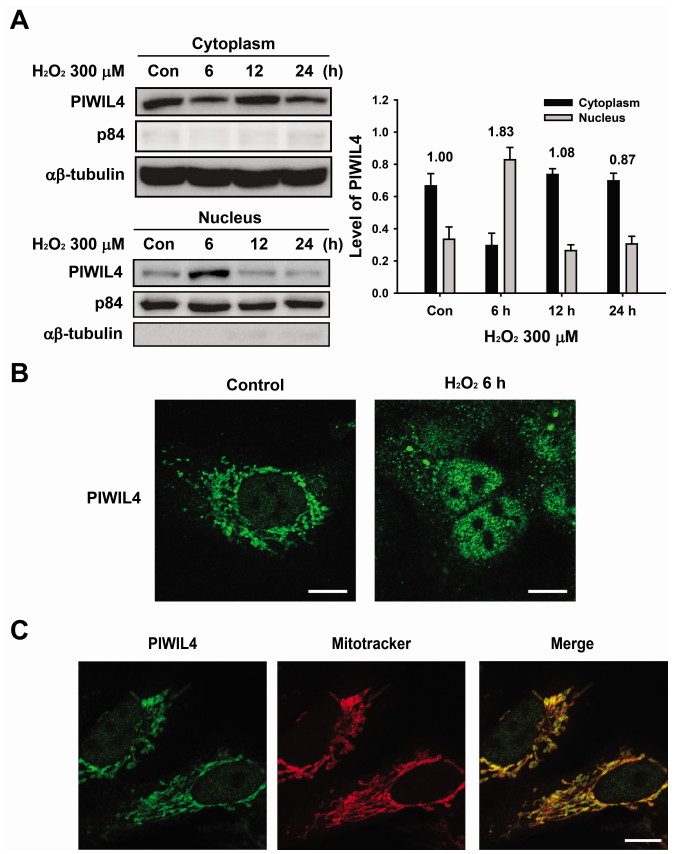

Fig. 2. Levels of PIWIL4 were increased in the nucleus during the early phase of oxidative stress. (A) Western blot analysis of PIWIL4 isolated from cytoplasmic and nuclear fractions in ARPE-19 cells treated with $\mathrm{H}_{2} \mathrm{O}_{2}(300 \mu \mathrm{M})$ for 6,12 , and $24 \mathrm{~h}$. Bar graph indicates fractions of PIWIL4 present in the cytoplasm and nucleus, which were normalized to $\alpha \beta$-tubulin (cytoplasmic marker) and p84 (nuclear marker). The numbers on the top of the quantitative bar graph represents the total amount of PIWIL4 normalized to the control (i.e. Con). (B) Fluorescence microscopy images of PIWIL4 (green fluorescence) in ARPE-19 cells treated with $\mathrm{H}_{2} \mathrm{O}_{2}(300 \mu \mathrm{M})$ for $6 \mathrm{~h}$. Scale bar: $10 \mu \mathrm{m}$. (C) Fluorescence images representing co-localization of PIWIL4 (green fluorescence) with mitochondria stained with Mitotracker (red fluorescence) in control cells. Scale bar: $10 \mu \mathrm{m}$. 
protein similar to PIWI, aggregated into SGs under oxidative stress with $\mathrm{NaAsO}_{2}$, which led to the obstruction of RNA cleavage activity of Ago2 (28). Moreover, most proteins that accumulate in SGs have RNA-binding function (29). Hence, we hypothesized that prolonged exposure of oxidative stress may lead to sequestration of PIWIL4 proteins into SGs. To test this hypothesis, SGs were fluorescently stained using an antibody against the SG-specific marker TIA1 in ARPE-19 cells that were treated with $\mathrm{H}_{2} \mathrm{O}_{2}$ for $24 \mathrm{~h}$ (Fig. 3A). When the cells were exposed to $\mathrm{H}_{2} \mathrm{O}_{2}$ for $24 \mathrm{~h}$, SGs were formed that showed TIA1 in an aggregated granular form with enhanced fluorescence. Importantly, PIWIL4 co-localized with TIA1 as shown by its fluorescence with a similar shape and distribution as the stress granules. When the $\mathrm{H}_{2} \mathrm{O}_{2}$ treated cells were co-treated with $\mathrm{CHX}$, which blocks formation of SGs, fluorescence of TIA1 showed a reduced aggregated form with reduced intensity, and PIWIL4 signal was mainly detected in the nucleus. The percentage of cells with PIWIL4 co-localized with SGs showed a steady increase up to $80 \%$ as the cells were continuously exposed to $\mathrm{H}_{2} \mathrm{O}_{2}$ up to $24 \mathrm{~h}$ (Fig. 3B). Interestingly, accumulation of PIWIL4 in the nucleus was only
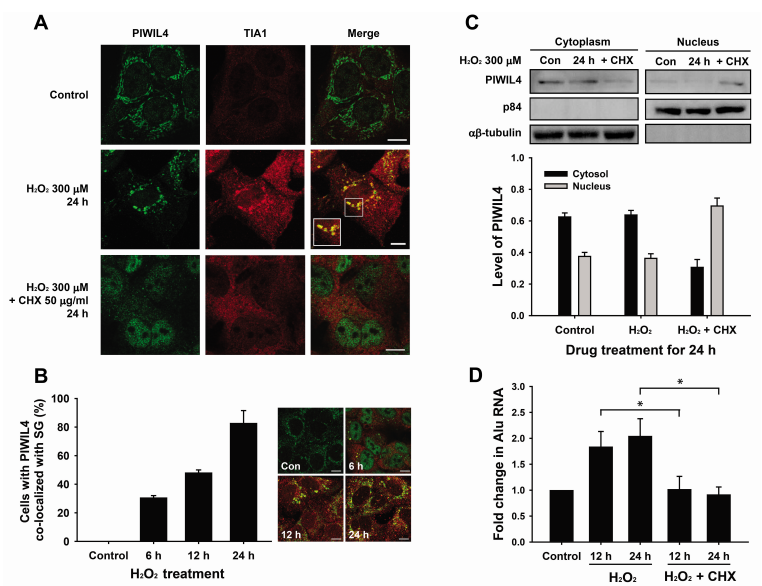

Fig. 3. Sequestration of PIWIL4 into stress granule impedes silencing of Alu RNA transcription. (A) Co-localization of PIWIL4 (green fluorescence) with TIA1 (stress granule marker; red fluorescence) in ARPE-19 cells treated with $\mathrm{H}_{2} \mathrm{O}_{2}(300 \mu \mathrm{M})$ in the absence or presence of cycloheximide $(\mathrm{CHX})(50 \mu \mathrm{g} / \mathrm{ml})$ for $24 \mathrm{~h}$; scale bar: $10 \mu \mathrm{m}$. (B) ARPE-19 cells were treated $300 \mu \mathrm{M} \mathrm{H} \mathrm{H}_{2} \mathrm{O}_{2}$ for 6,12 , and $24 \mathrm{~h}$, and immunostained with PIWIL4 and TIA1. The bar graph indicates the percentage of cells expressing PIWIL4 that co-localized with stress granule. (C) Western blot analysis of PIWIL4 isolated from cytoplasmic and nuclear fractions in ARPE-19 treated with $\mathrm{H}_{2} \mathrm{O}_{2}(300 \mu \mathrm{M})$ in the absence or presence of $\mathrm{CHX}(50 \mu \mathrm{g} / \mathrm{ml})$ for $24 \mathrm{~h}$. Bar graph indicates fractions of PIWIL4 present in the cytoplasm and nucleus, which were normalized to $\alpha \beta$-tubulin and p84. (D) ARPE-19 cells were treated with $\mathrm{H}_{2} \mathrm{O}_{2}(300 \mu \mathrm{M})$ for 12 and $24 \mathrm{~h}$ in the absence or presence of $\mathrm{CHX}(50 \mu \mathrm{g} / \mathrm{ml})$. After incubation, total RNA was obtained and amplified to cDNAs. Alu RNAs were relatively quantified by real-time PCR and normalized to the control cells. Data are presented as the \pm S.E.M. $n=3$. $* P<0.05$. observed in the early phase (i.e., 6 h) of ROS exposure, consistent with the preceding result (Fig. 2).

To further clarify the unequal distribution of PIWIL4 in the nucleus and cytoplasm, the subcellular presence of PIWIL4 in the cells treated with $\mathrm{H}_{2} \mathrm{O}_{2}$ with or without $\mathrm{CHX}$ for $24 \mathrm{~h}$ were analyzed with western blot analysis of nuclear and cytoplasmic fractions (Fig. 3C). Consistent with the immunocytochemistry results (Fig. 3A), PIWIL4 was mainly present in the cytoplasm under the prolonged oxidative stress condition (24 h). However, the nuclear localization of PIWIL4 was significantly diminished with $\mathrm{CHX}$ co-treatment, suggesting that PIWIL4 localization in the cytoplasm depended on SG formation. Thus, the subcellular distribution of PIWIL4 was governed by SG formation, by which PIWIL4 was sequestrated into SGs under prolonged oxidative stress conditions.

Next, we monitored the levels of Alu RNA expression in the

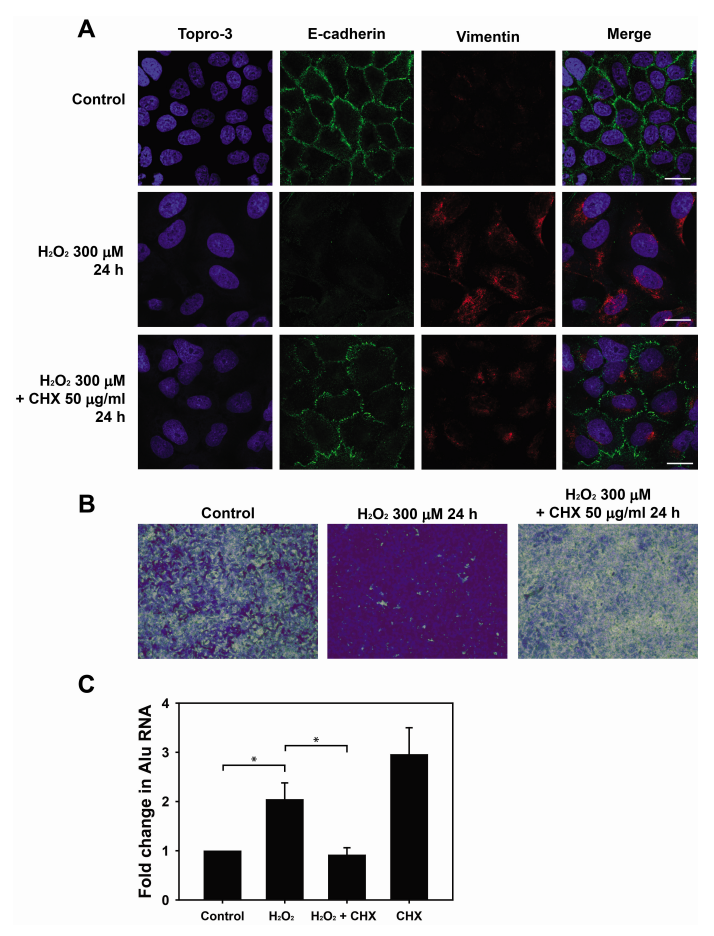

Fig. 4. Oxidative stress induces upregulation of $A l u$ RNA as well as epithelial-to-mesenchymal transition in RPE cells. (A) ARPE-19 cells were treated $\mathrm{H}_{2} \mathrm{O}_{2}(300 \mu \mathrm{M})$ in the absence or presence of cycloheximide $(\mathrm{CHX}) \quad(50 \mu \mathrm{g} / \mathrm{ml})$ for $24 \mathrm{~h}$, and subsequently immunostained for E-cadherin (green fluorescence) and vimentin (red fluorescence). The nuclei were visualized with DAPI (blue fluorescence); scale bar: $20 \mu \mathrm{m}$. (B) Cell migration assay of ARPE-19 cells after $\mathrm{H}_{2} \mathrm{O}_{2}(300 \mu \mathrm{M})$ treatment in the absence or presence of $\mathrm{CHX}(50 \mu \mathrm{g} / \mathrm{ml})$ for $24 \mathrm{~h}$ using a transwell. Migrated cells were stained with crystal violet. (C) The bar graph represents the relative amounts of $A / u$ RNAs normalized to GAPDH in ARPE-19 cells treated with $\mathrm{H}_{2} \mathrm{O}_{2}(300 \mu \mathrm{M})$ in the absence or presence of $\mathrm{CHX}(50 \mu \mathrm{g} / \mathrm{ml})$ for $24 \mathrm{~h}$. Data are presented as the \pm S.E.M. $\mathrm{n}=3 .{ }^{*} \mathrm{P}<0.05$. 
cells treated with $\mathrm{H}_{2} \mathrm{O}_{2}$ with or without $\mathrm{CHX}$ for $24 \mathrm{~h}$ (Fig. 3D). As compared to the control, levels of Alu RNA transcripts significantly increased with prolonged exposure of $\mathrm{H}_{2} \mathrm{O}_{2}$ up to $24 \mathrm{~h}$. However, the increase of Alu RNA expression was not observed in the cells co-treated $\mathrm{H}_{2} \mathrm{O}_{2}$ and $\mathrm{CHX}$ for $24 \mathrm{~h}$, which shows a similar level to untreated cells. This result demonstrates that inhibition of SG formation causes suppression of Alu RNA transcription in the RPE cells under prolonged oxidative stress conditions. Taken together, these data suggest that accumulation of Alu RNAs was likely caused by an entrapment of PIWIL4 into SGs, resulting in a failure in the epigenetic silencing of Alu RNA transcription by PIWIL4.

\section{Accumulation of Alu RNA was correlated with EMT transition of RPE cells under oxidative stress condition}

Since Alu RNA has been observed to be related with EMT in cancer cells (14), we next investigated whether augmented expression of Alu RNAs was correlated with EMT in RPE cells. To monitor the progression of EMT in the cells under oxidative stress, the expression of E-cadherin and vimentin, markers of EMT, were examined by immunocytochemistry (Fig. 4A). Control RPE cells maintained a high level of well-structured E-cadherin and low expression of vimentin, showing a sustained epithelial feature. However, prolonged exposure of $\mathrm{H}_{2} \mathrm{O}_{2}$ induced downregulation of E-cadherin and overexpression of vimentin, representing the progression of EMT. Importantly, when the cells were co-treated with $\mathrm{H}_{2} \mathrm{O}_{2}$ and $\mathrm{CHX}$, mesenchymal transition was significantly diminished as shown by the intact epithelial E-cadherin and reduced expression of vimentin.

In addition, we performed a transwell migration assay to examine the migratory properties of RPE cells that were undergoing the EMT process under the oxidative stress condition (Fig. 4B). Migrated cells were stained with crystal violet, and migratory effects were compared with the control group. The cells treated with $\mathrm{H}_{2} \mathrm{O}_{2}$ for $24 \mathrm{~h}$ showed enhanced migratory properties, as compared to the control cells without $\mathrm{H}_{2} \mathrm{O}_{2}$ treatment. In contrast, when the cells were co-treated with $\mathrm{H}_{2} \mathrm{O}_{2}$ and $\mathrm{CHX}$, cellular migration was significantly diminished. This result was consistent with the EMT progression, in which oxidative stress causes mesenchymal transition and migration of RPE cells. However, the mesenchymal transition of the RPE cells was significantly attenuated by the $\mathrm{CHX}$ treatment, which blocks the formation of SGs that sequester PIWIL4, and subsequent downregulation of Alu RNA transcription (Fig. 3).

To test whether accumulation of Alu RNA is correlated with EMT of RPE cells, the amount of Alu RNAs in RPE cells under the same conditions as above was quantified and compared (Fig. 4C). When the cells were treated with $\mathrm{H}_{2} \mathrm{O}_{2}$ for $24 \mathrm{~h}$, approximately 2 -fold increase of Alu RNA expression was observed as compared to the control. Consistent with the previous result (Fig. 3D), Alu RNA expression was not enhanced in the cells co-treated with $\mathrm{H}_{2} \mathrm{O}_{2}$ and $\mathrm{CHX}$. These results suggest that accumulation of Alu RNA was positively correlated with the progression of mesenchymal transition of RPE cells under prolonged oxidative stress, resulting in loss of cell-cell tight junctions and subsequent cellular migration.

In conclusion, the present study demonstrated that the temporal and spatial distribution of PIWIL4 altered levels of Alu RNAs in RPE cells under oxidative stress. We found that PIWIL4 mainly localized to the nucleus and subsequently downregulated the expression of Alu RNAs during the early phase of ROS exposure. As the oxidative stress continued, PIWIL4 was sequestered into cytoplasmic stress granules, resulting in a failure of epigenetic silencing of Alu RNA transcription in the nucleus and an accumulation of Alu RNA. When the formation of stress granules was inhibited, the nuclear localization of PIWIL4 was restored and the expression of Alu RNAs was attenuated. In addition, an elevated amount of Alu RNA was positively correlated with a disruption of the epithelial features of RPE cells via induction of mesenchymal transition. Thus, we propose that oxidative stress causes Alu RNA accumulation via PIWIL4 sequestration into the cytoplasmic stress granule. Furthermore, our study suggests that inhibition of stress granule formation may relieve the mesenchymal transition of RPE cells under oxidative stress by allowing PIWIL4 to downregulate expression of Alu RNA.

\section{MATERIALS AND METHODS}

Detailed materials and methods are available in Supplementary Data.

\section{ACKNOWLEDGEMENTS}

This research was supported by the National Research Foundation of Korea funded by the Ministry of Science and ICT, Republic of Korea (NRF-2017R1E1A1A01074656).

\section{CONFLICTS OF INTEREST}

The authors have no conflicting interests.

\section{REFERENCES}

1. Szmulewicz MN, Novick GE and Herrera RJ (1998) Effects of Alu insertions on gene function. Electrophoresis 19, 1260-1264

2. Kazazian HH Jr (2004) Mobile elements: drivers of genome evolution. Science 303, 1626-1632

3. Muotri AR, Marchetto MC, Coufal NG and Gage FH (2007) The necessary junk: new functions for transposable elements. Hum Mol Genet 16 Spec No. 2, R159-167

4. Batzer MA and Deininger PL (2002) Alu repeats and human genomic diversity. Nat Rev Genet 3, 370-379

5. Deininger PL and Batzer MA (1999) Alu repeats and human disease. Mol Genet Metab 67, 183-193

6. Wallace MR, Andersen LB, Saulino AM, Gregory PE, 
Glover TW and Collins FS (1991) A de novo Alu insertion results in neurofibromatosis type 1. Nature 353, 864-866

7. Komkov A, Maschan MA, Shvets VI and Lebedev lu B (2012) [The functional analysis of polymorphic insertions of Alu retroelements at acute lymphoblastic leukemia]. Bioorg Khim 38, 351-364

8. Wu SJ, Hsieh TJ, Kuo MC et al (2013) Functional regulation of Alu element of human angiotensin-converting enzyme gene in neuron cells. Neurobiol Aging 34, 1921 e1921-1927

9. Fazza AC, Sabino FC, de Setta N, Bordin NA Jr, da Silva EH and Carareto CM (2009) Estimating genomic instability mediated by Alu retroelements in breast cancer. Genet Mol Biol 32, 25-31

10. Li TH and Schmid CW (2001) Differential stress induction of individual Alu loci: implications for transcription and retrotransposition. Gene 276, 135-141

11. Liu WM, Chu WM, Choudary PV and Schmid CW (1995) Cell stress and translational inhibitors transiently increase the abundance of mammalian SINE transcripts. Nucleic Acids Res 23, 1758-1765

12. Kaneko H, Dridi S, Tarallo V et al (2011) DICER1 deficit induces Alu RNA toxicity in age-related macular degeneration. Nature 471, 325-330

13. Tarallo V, Hirano Y, Gelfand BD et al (2012) DICER1 loss and Alu RNA induce age-related macular degeneration via the NLRP3 inflammasome and MyD88. Cell 149, 847-859

14. Di Ruocco F, Basso V, Rivoire M et al (2018) Alu RNA accumulation induces epithelial-to-mesenchymal transition by modulating miR-566 and is associated with cancer progression. Oncogene 37, 627-637

15. Hadjiargyrou $M$ and Delihas $N$ (2013) The intertwining of transposable elements and non-coding RNAs. Int J Mol Sci $14,13307-13328$

16. Aravin A, Gaidatzis D, Pfeffer $S$ et al (2006) A novel class of small RNAs bind to MILI protein in mouse testes. Nature 442, 203-207

17. Su C, Ren ZJ, Wang F, Liu M, Li X and Tang H (2012)
PIWIL4 regulates cervical cancer cell line growth and is involved in down-regulating the expression of p14ARF and $\mathrm{p53}$. FEBS Lett 586, 1356-1362

18. Liu X, Sun Y, Guo J et al (2006) Expression of hiwi gene in human gastric cancer was associated with proliferation of cancer cells. Int J Cancer 118, 1922-1929

19. Zhao YM, Zhou JM, Wang LR et al (2012) HIWI is associated with prognosis in patients with hepatocellular carcinoma after curative resection. Cancer 118, 2708-2717

20. Sivagurunathan S, Palanisamy K, Arunachalam JP and Chidambaram S (2017) Possible role of HIWI2 in modulating tight junction proteins in retinal pigment epithelial cells through Akt signaling pathway. Mol Cell Biochem 427, 145-156

21. Ambati J and Fowler BJ (2012) Mechanisms of age-related macular degeneration. Neuron 75, 26-39

22. Manning BD and Cantley LC (2007) AKT/PKB signaling: navigating downstream. Cell 129, 1261-1274

23. Sadidi M, Lentz SI and Feldman EL (2009) Hydrogen peroxide-induced Akt phosphorylation regulates Bax activation. Biochimie 91, 577-585

24. Baek A, Yoon S, Kim J et al (2017) Autophagy and KRT8/keratin 8 protect degeneration of retinal pigment epithelium under oxidative stress. Autophagy 13, 248-263

25. Le Thomas A, Toth KF and Aravin AA (2014) To be or not to be a piRNA: genomic origin and processing of piRNAs. Genome Biol 15, 204

26. Anderson $P$ and Kedersha N (2009) RNA granules: post-transcriptional and epigenetic modulators of gene expression. Nat Rev Mol Cell Biol 10, 430-436

27. Anderson P and Kedersha N (2008) Stress granules: the Tao of RNA triage. Trends Biochem Sci 33, 141-150

28. Detzer A, Engel C, Wunsche W and Sczakiel G (2011) Cell stress is related to re-localization of Argonaute 2 and to decreased RNA interference in human cells. Nucleic Acids Res 39, 2727-2741

29. Buchan JR and Parker R (2009) Eukaryotic stress granules: the ins and outs of translation. Mol Cell 36, 932-941 